\section{Health technology assessment processes for nanotechnologies: the ethical domain}

\author{
Antonio G. Spagnolo, Pietro Refolo, \\ Dario Sacchini, Viviana Daloiso \\ Institute of Bioethics, A. Gemelli School \\ of Medicine, Università Cattolica del \\ Sacro Cuore, Rome, Italy
}

\section{Abstract}

The ethical assessment of the use of technologies is generally considered a component of the health technology assessment (HTA) processes. HTA is a multidisciplinary process that summarizes information about medical, economic, organizational, ethical, psychological, social and legal issues related to the implementation of a certain health technology in health care system and its main purpose is to inform policymaking. Unlike the other technologies nanotechnologies pose different risks and, therefore, new bioethical implications should be assessed. So, the ethical assessment of nanotechnologies within the HTA processes could be more problematic. The article intends to debate this complexity.

\section{What is health technology assess- ment?}

According to the International Network of Agencies for Health Technology Assessment (INAHTA), Health Technology Assessment (HTA) is the systematic evaluation of properties, effects, andor impacts of health care technology. It may address the direct, intended consequences of technologies as well as their indirect, unintended consequences. Its main purpose is to inform technology-related policymaking in health care. HTA is conducted by interdisciplinary groups using explicit analytical frameworks drawing from a variety of methods. ${ }^{1}$ In other words, HTA is a multidisciplinary process that summarizes information about medical, economic, organizational, ethical, psychological, social and legal issues related to the implementation of a certain health technology in health care system and its main purpose is to inform policymaking.

At the present, it may represent the more functional support available for supporting decisions that regard the introduction and/or delisting use of health technologies. It is important to point out that the term health technology does not only refer to tangible devices: in fact, it covers a wide range of methods of intervening to promote health, including the prevention, diagnosis or treatment of disease, the rehabilitation or long-term care of patients, as well as drugs, devices, clinical procedures and healthcare settings. ${ }^{1}$

Moreover, HTA may involve the investigation of one or more properties, impacts, or other attributes of health technologies or applications. In general, these include the following: technical properties; safety; efficacy and/or effectiveness; economic attributes or impacts; social, legal, ethical and/or political impacts.

Finally, there is great variation in the scope, selection of methods and level of detail in the practice of HTA. Nevertheless, most HTA activity involves some form of the following basic steps: i. identify assessment topics; ii. specify the assessment problem; iii. determine locus of assessment; iv. retrieve evidence; v. collect new primary data (as appropriate); vi. appraise/interpret evidence; vii. integrate/synthesize evidence; viii. formulate findings and recommendations; ix. disseminate findings and recommendations; $\mathrm{x}$. monitor impact.

\section{The ethical assessment within health technology assessment processes}

HTA has always welcomed the idea of an ethical assessment for health technologies.

This type of assessment should pose the ethical questions raised by the technology itself and by the consequences of implementing/not implementing a health technology as well as the ethical issues that are inherent in the HTA process. ${ }^{2,3}$ Although the ethical assessment is listed as one of its purposes, the integration of this domain into HTA reports has often been limited. ${ }^{3,4}$

The reasons are several: i. technologies are often considered by HTA producers as being ethically neutral and value-free; ii. the only technical and economical questions are perceived as important; iii. the training of HTA producers and available resources to conduct ethical analyses are often limited; iv. there is a wide heterogeneity of research methodologies. $^{5-7}$ With particular reference to point iv. HTA analyses are generally realized by methods and criteria standardized among countries or HTA agencies, while the ethical assessment depends on the ethical criteria and methodologies of the different schools where they are formulated. The European Network for Health Technology Assessment (EUnetHTA) has conducted a survey (EUnetHTA Project 2006-2008) on all research methodologies currently adopted by HTA agencies all over the world. It has underlined a wide range of possibilities: up till now the more widespread methodologies are Casuistry, Coherence analysis, Interactive, participatory HTA approach, Principlism, Social shaping of technology and Wide reflective equilibrium, beyond local approaches - as the EUnetHTA has defined them - like, for
Correspondence: Antonio G. Spagnolo,

Istituto di Bioetica, Facoltà di Medicina e chirurgia, A. Gemelli, Università Cattolica del Sacro Cuore, Largo F. Vito, 1 - 00168 Roma.

Tel. +39.06.30156007 - +39.06.30155861 -

Fax: +39063051149 .

E-mail: agspagnolo@rm.unicatt.it

Key words: health technology assessment, nanotechnology, bioethics.

Acknowledgments: the authors would like to thank the two anonymous reviewers for helpful comments and suggestions.

Contributions: the authors contributed equally.

Conflict of interest: the authors report no conflicts of interest.

Received for publication: 15 April 211.

Accepted for publication: 29 July 2011.

This work is licensed under a Creative Commons Attribution NonCommercial 3.0 License (CC BYNC 3.0).

(C) Copyright A.G. Spagnolo et al., 2011 Licensee PAGEPress, Italy

Nanotechnology Development 2011; 1:e1 doi:10.4081/nd.2011.e1

example, the Triangular model or the Eclectic approach.

The heterogeneity of approaches certainly makes more difficult the already thorny ethicsHTA relationship. So, the EUnetHTA Project has made an attempt to provide a common basis for ethical evaluation and it has elaborated a model, that has two versions, one for medical and surgical interventions, the other for diagnostic technologies. ${ }^{8,9}$ At present, the European network on HTA is going on as EUnetHTA Joint Action 2011-2012, working about models on genetic screening and on Relative Effectiveness Assessment of pharmaceuticals. This model - as Saarni et al. have observed - does not purport to solve the philosophical debate but to offer a tool usable by HTA organizations, irrespective of their resources (material, time and knowledge). ${ }^{7}$

In short, it consists of three elements: a set of questions that concern the fundamental issues for ethical assessment; a description of methodologies by which the different issues could be approached; and the debate on the process of integration of the ethical assessments into HTA reports.

First attempts of application of the EUnetHTA model seem to be encouraging. ${ }^{10,11}$ Nevertheless, the assessment of nanotechnologies may represent a very interesting challenge for it. 
Health technology assessment and nanotechnologies

Nanotechnologies may be outlined as the expression of the human ability to manipulate atoms and to build - thanks to the properties typical of the size (nanometric scale) - devices and materials with novel properties and functions. According to the US National Nanotechnology Initiative (NNI), nanotechnology involves research and technology development at the atomic, molecular or macromolecular levels, in the length scale of approximately 1 to $100 \mathrm{~nm}$ range, to provide a fundamental understanding of phenomena and materials at the nanoscale and to create and use structures, devices, and systems that have novel properties and functions because of their small size and/or intermediate size. ${ }^{12}$

Others define nanotechnologies looking at the opportunities deriving from their employment or identifying tools and devices typical of the nanometric scale.

Nanotechnologies have been investigated from several fields; even though medicine, where they are known as nanomedicine, is the field where there is a greater interest in, moving from prevention to diagnosis and treatment. In particular, nanotechnologies introduce novel therapies (the form of drug delivery), new drugs (drugs discovery) and new tools and devices (nanomaterials, nanodevices). From this point of view, nanotechnologies can be considered as health technologies and, therefore, submitted to an HTA process.

Being also a convergence of many other disciplines (for example, from physics to engineering computer science), nanotechnologies open to several opportunities, but, at the same time, they contribute to enlarge the circumference of the unknown, as Bartha Knoppers has hypothesized: as the radius of knowledge gets longer, the circumference of the unknown increases even more. ${ }^{13}$

So, some authors consider the ethical issues that may arise from these technologies Apocalyptic Nightmares or Utopian Dreams..$^{14,15}$

Furthermore, they open to debate between those who favour the need for a specific ethical evaluation (called nano-ethics) and those for whom nanotechnologies generate no new bioethical issues and refer to these technologies as old bioethical issues (distributive justice, ethics of research, to mention only these). The question lies perhaps in the way the concept nano-ethics is interpreted: ${ }^{16}$ those who do not share the need for a specific bioethics around nanotechnologies base their considerations on the fact that these technologies do not produce a change of values and do not require the introduction of new ethical principles. At a closer look, nanoethics does not introduce new principles or values, but some implications are of course specific to nanotechnologies: compared with other tech- nologies, they have different objectives and, above all, pose different risks that come from some of their peculiar characteristics.

This makes their assessment within the HTA processes more problematic, in particular for what concerns safety, efficacy and the ethical aspects.

Safety regards the assessment of the acceptability of a given risk both for the patient and for those who are involved in the use of the technology. As it is known, toxicity represents a peculiar aspect of these technologies. This can be said both for exposure from ingestion of nanoproducts (in the form of drug delivery devices) and from nanodevices implanted in the human body. In the first case, while some modalities of exposure are known, above all skin absorption and inhalation, with the possibility to follow their translocation and distribution in the body, many others remain unknown. As far as it regards nanoparticles' distribution and their toxicity, a recent opinion of the US Food and Drug Administration (FDA) refers that studies have (also) shown that modifying the surface of nanoscale materials with surfactants or biocompatible polymers reduced the toxicity in vitro and altered the half-life and tissue deposition in vivo. ${ }^{17}$ For this reason, it is difficult to determine the toxicity profiles of many nanoparticles for risk assessment. ${ }^{18-20}$

Efficacy refers to the benefit, in terms of health, rising from the use of the technology in question. Under this aspect, nanotechnologies are very promising and useful technologies because of the properties coming from size. In particular, thanks to these novel properties, it is possible to achieve the therapeutic efficacy with less invasive technique and less devastating side effects induced, for example, by chemotherapy drugs, or during diagnosis it will be possible to localize, with precision, the site of the disease and, at the same time, deliver the appropriate drug, in the right dosage.

It is unquestionable that the possibilities of combining science and technologies to create more tailored and effective therapies represent a benefit for the patient. Nevertheless, these benefits should be interpreted in the light of the risk associated with the treatment. Efficacy of nanotechnology is hard to be defined, due to the incertitude associated with nanoparticles. In particular, due to their very small size, nanoparticles are small enough to enter cells in organisms, cross cell membranes, move to organs and tissues allowing a targeted distribution of drugs. At the same time, the size itself of these particles may create risks: it has been shown that often materials non-toxic in their natural state, at the atomic magnitude became toxic. For example, carbon nanotubes may largely be employed in clinical practice; even though studies have shown that the biological behavior of these particles may produce reactive oxygen species
(ROS) - free radicals -, mitochondrial dysfunction (to cite only these). ${ }^{21}$ More specificly - as stated by FDA - nanoscale materials often have chemical, physical, or biological properties that are different from those of their larger counterparts. Such differences may include altered magnetic properties, altered electrical or optical activity, increased structural integrity or altered chemical or biological activity. ${ }^{18}$ This is primarily due to the high-surface-tomass relation of nanoparticles: because most of the chemical reactions take place on the surface, the smaller the particles are, as its structure becomes finer, the bigger the masssurface ratio, so that the greater percentage of the atoms lies on the surface and the material become more reactive. This incremented surface may modify particles generally not very active into active nanoparticles. Many experimental studies on nanomaterials, carried out on rats, have shown that, mass being equal, insoluble nanoparticles are more toxic than particles of a larger size of similar composition; in particular the side effect is given by the inflammation of the pulmonary tissue and by tumors. ${ }^{22,23}$ Given the same material, nanoengineered particles show an incremented biological activity compared to that shown by particles of greater size.

\section{The ethical domain in the health technology assessment process for nanotechnologies}

The same characteristics that make the use of nanotechnologies so promising from a medical point of view make it ticklish from an ethical point of view. Specifically, beyond the above-mentioned issues related to the novel properties that have consequences for the assessment of safety and efficacy, nanotechnologies raise, among others, the following ethical questions: ${ }^{24}$ i) Nanotechnologies make difficult the identification of a systematic toxicological risk assessment/management of nanomaterials and products. ${ }^{25,26}$ This lack raises further issues for the subjects' informed consent within clinical trials, which are more complex because of the lack of in vivo study in animals; ii) It is difficult to define, at least in the short term, all the implications (for example, harms) deriving from these novel technologies; iii) Nanotechnologies, due to their novelty, may produce a gap between scientific research and foreseen outcomes; iv) Many nanotechnologies are home-care technology: this could cause the depersonalization of many physician-patient relationships, causing changes in the way the medical profession is practiced; v) Managing information and privacy is an ongoing problem: in other words, there is the worry that nanobiotechnologies, able to reduce dimensions and increase functions, may be used for other than medical purposes, 
for example, to monitor human life and personal privacy. Moreover, it could be difficult to store the large amount of data coming from these devices; vi) Another ethical issue deals with the consideration for which diagnostic nanotechnologies may produce incidental findings of pathologies that still have unexplained symptoms. Such findings may create a need for patients and clinicians to clarify their clinical relevancy. In other words, diagnostic investigation of incidental findings may require further investigations. This is a thorny since it could often force patients into additional diagnostic investigations with multiple consequences; vii) nanotechnologies may be used to enhance, repair or replace human characteristics. In the light of these points it seems plain that new strategies for the ethical assessment of nanotechnologies within HTA processes should be planned. They should at least involve the assessment of adequacy between the introduction/use of technologies and the good of a person. In other words, verification of their use realizes this good. Some of us have previously described in $\operatorname{detail}^{3}$ a process of ethical evaluation within HTA, considering three steps, as the vertices of an ideal triangle: i. data collection (cognitive level); ii. ethical analysis (justifying level); iii. ethical evaluation and deliberation (deliberative level). The first step (point $A$ of the triangle) is an in-depth study of all factual data. In order to realize this analysis, putting these questions could be useful: i) what is it about? ii) how is it to be done? iii) why is it done? iv) what are the consequences? The second step (point B) is the anthropological and ethical understanding of facts that is the analysis of values at stake or in conflict. To achieve this evaluation, following operating criteria are utilized: i) the respect for human physical life; ii) the contextual exercise of freedom and responsibility within decision-making process; iii) the safeguard of the therapeutic principle, according to which the human person has to be treated as a whole of body and soul; iv) the principles of sociality and subsidiarity. The third step (point C) consists of the ethical evaluation that guides the practical choice.

\section{Conclusions}

The implementation of new tools, like nanotechnologies, into clinical research could at the same time represent a risk and an opportunity for human subjects. Challenges issued by nanotechnologies consist, above all, of the risk assessment. Risk depends on many factors, like size, shape, novel properties of these particles, typical elements of the magnitude, nanometric scale, and then on the novel behavior of nanoparticles, that could be not foresee- able and are hard to investigate by the traditional evaluation criteria. This is a challenge, however, not impossibility. So, new strategies for the ethical assessment of nanotechnologies within HTA processes shall be planned, taking into account the peculiar elements of these enhancing technologies.

\section{References}

1. International Network of Agencies for Health Technology Assessment (INAHTA). Health Technology Assessment (HTA) Glossary. Available from: http://www.inahta.org/G0-DIRECT-TO/Members/

2. Hofmann BM. Why ethics should be part of health technology assessment. Int $\mathrm{J}$ Technol Assess Health Care 2008;24:423-9.

3. Sacchini D, Virdis A, Refolo P, et al. Health technology assessment (HTA): ethical aspects. Med Health Care Philos 2009; 12:453-7.

4. Hofmann B. Toward a procedure for integrating moral issues in health technology assessment. Int $\mathrm{J}$ Technol Assess Health Care 2005;21:312-8.

5. ten Have H. Medical technology assessment and ethics. Ambivalent relations. Hastings Cent Rep 1995;25:13-9.

6. ten Have H. Ethical perspectives on health technology assessment. Int $\mathrm{J}$ Technol Assess Health Care 2004;20:71-6.

7. Saarni SI, Hofmann B, Lampe K, et al. Ethical analysis to improve decision-making on health technologies. Bull World Health Organ 2008;86:617-23.

8. Lampe K, Mäkelä M; EUnetHTA WP4. HTA Core Model for Medical and Surgical Interventions - Version 1.0R. Available from: http://www.Eunethta.net/upload/ WP4/Final\%20Deliverables/HTA\%20Core\% 20Model\%20for\%20Medical\%20and\%20Su rgical\%20Interventions\%201\%200r.pdf

9. Mäkelä M, Pasternack I, Lampe K; EUnetHTA WP4. Core HTA on drug eluting stents (Des). Available from: http://www. eunethta.net/upload/WP4/Final\%20Deliver ables/Core\%20HTA\%20on\%20Drug\%20Elu ting\%20Stents.pdf

10. Pasternack I, Anttila H, Mäkelä M, et al. Testing the HTA core model: experiences from two pilot projects. Int $\mathrm{J}$ Technol Assess Health Care 2009;25 Suppl 2:21-7.

11. Pasternack I, Lampe K; EUnetHTA WP4. Core HTA on Msct Coronary Angiography. Available from: http://www.eunethta.net/ upload/WP4/Final\%20Deliverables/Core\%2 0HTA\%20on\%20MSCT\%20Angiography.pdf

12 Available from: http://www.nano.gov/ html/facts/whatIsNano/html

13. Collins FS, McKusick VA. Implications of the Human Genome Project for medical science. JAMA 2001;285:540-44.

14. Gordijin B. Nanoethics: from utopian dreams and apocalyptic nightmares toward a more balanced view. Sci Eng Ethics 2005;11:521-33.

15. Maynard AD. Nanotechnology: the next big thing, or much ado about nothing? Ann Occup Hyg 2007;51:1-12.

16. Spagnolo AG, Daloiso V. Outlining ethical issues in nanotechnologies. Bioethics 2009;23:394-402.

17. Food and Drug Administration, Nanotechnology Task Force. Nanotechnlogy. Available from: http://www.fda.gov/nanotechnology/taskforce/report2007.html

18. Hoet PH, Brüske-Hohlfeld I, Salata OV. Nanoparticles - known and unknown health risks. J Nanobiotechnology 2004;2: 12-25.

19. Oberdörster G, Maynard A, Donaldson K, et al. Principles for characterizing the potential human health effects from exposure to nanomaterials: elements of a screening strategy. Part Fibre Toxicol 2005;2:8.

20. Gwinn MR, Vallyathan V. Nanoparticles: health effects -- pros and cons. Environ Health Perspect 2006;114:1818-25.

21. Borm JA, Müller-Schulte D. Nanoparticles in drug delivery and environmental exposure: same size, same risks? Nanomedicine (Lond) 2006;1:235-49.

22. 2nd International Symposium on Nanotechnology and occupational health. Available from: http://www.cce.umn.edu/ pdfs/cpe/conferences/nanotech_abstract.pdf

23. Oberdörster G, Oberdörster E, Oberdörster J. Nanotoxicology: an emerging discipline evolving from studies of ultrafine particles. Environ Health Perspect 2005;113: 823-39.

24. Daloiso V, Refolo P, Spagnolo AG. La valutazione etica delle nanotecnologie nei processi di HTA. In: Ruggiu D, Pariotti E, Guerra G, Piccinni M. Regolamentazione e responsabilità nelle nanotecnologie. Bologna: Il Mulino. 2011 (in press).

25. Scientific Committee on Emerging and Newly Identified Health Risks (SCENHIR). Opinion on The Appropriateness of existing methodologies to assess the potential risks associated with engineered and adventitious products of nanotechnologies. 2006. Available from: http:/ec. europa.eu/health/ ph_risk/committees/04_scenihr/docs/scenih r_o_003b.pdf

26. Scientific Committee on Emerging and Newly Identified Health Risks (SCENHIR). Opinion on The Appropriateness of the Risks Assessment Methodology in Accordance with the technical Guidance Documents for New and Existing substances for Assessing the Risks of Nanomaterials. Available from: http://ec.europa.eu/health/ph_risk/committees/04_scenihr/docs/scenihr_o_010.pdf 